\title{
Spiegelung von Seuchen in Öffentlichkeit und Kultur
}

\author{
Heinz-Peter Schmiedebach ${ }^{(\bowtie)}$ \\ Ehem. Stiftungsprofessor für Medical Humanities, Institut für Geschichte der \\ Medizin und Ethik in der Medizin, Charité Universitätsmedizin Berlin, Berlin, \\ Deutschland \\ heinz-peter.schmiedebachecharite.de
}

Schlüsselwörter: Seuchennarrativ $\cdot$ Sinnzuweisung $\cdot$ Politische

Instrumentalisierung $\cdot$ Tuberkulose $\cdot$ Grippepandemie $\cdot$ Pest $\cdot$ AIDS $\cdot$ COVID-

19-Pandemie

\section{Seuchennarrativ}

Wenn Staaten gezwungen sind, sich mit Pandemien auseinanderzusetzen, so werden primär biologische, medizinische, epidemiologische und ökonomische Aspekte in den Blick genommen. Seuchen sind aber auch soziale Ereignisse, was die Medizingeschichte, die Geschichte und die Kulturwissenschaften schon seit Jahrzehnten erforschen [1-7].

Seuchen werden in kommunikativen Prozessen erfahrbar und sie aktivieren künstlerische Kreativität. Besondere Metaphern und dramatische Bilder verleihen der Pandemie Gestalt. In dieser Kommunikation werden mannigfaltige Narrative entwickelt. Es geht um Helden und Schurken, um Opfer, Leid, Ängste verschiedenster Art und Solidarität, aber auch um Sinnsuche und Funktionalisierung. Epidemien und Pandemien werden also in sehr verschiedenen Sphären einer Gesellschaft erlebt und verarbeitet. Davon zeugt ein schier unermessliches Korpus an ikonografischen, literarischen, musikalischen, filmischen und anderen Produkten. Diese kulturellen Erzeugnisse gestatten uns einen Blick in die Vielfalt der Resonanzen, die ein Infektionsgeschehen in einer Gesellschaft hervorruft.

Als Beispiel sei hier eine bildliche Auseinandersetzung mit AIDS aus dem Jahr 1987 angeführt. Der Künstler Volker Bartsch schuf auf dem Höhepunkt der AIDSPandemie ein Bild, dessen Titel „Girl on the rocks“ an den Drink „Martini on the rocks" angelehnt ist (Abb. 1). Man sieht eine nackte Frau, die dem Betrachter zugewandt auf einem Haufen eiswürfelartiger, scharfkantiger Felsbrocken sitzt, aber jede Kontaktaufnahme in Anbetracht des eisigen, felsartigen Untergrunds unmöglich macht. Nach der sexuellen Libertinage der 1970er Jahre folgte in den 1980ern Jahren bedingt durch AIDS eine sexuelle Eiszeit. Die Kunst macht hier den eminenten Wandel im Sexualverhalten zum Thema. 


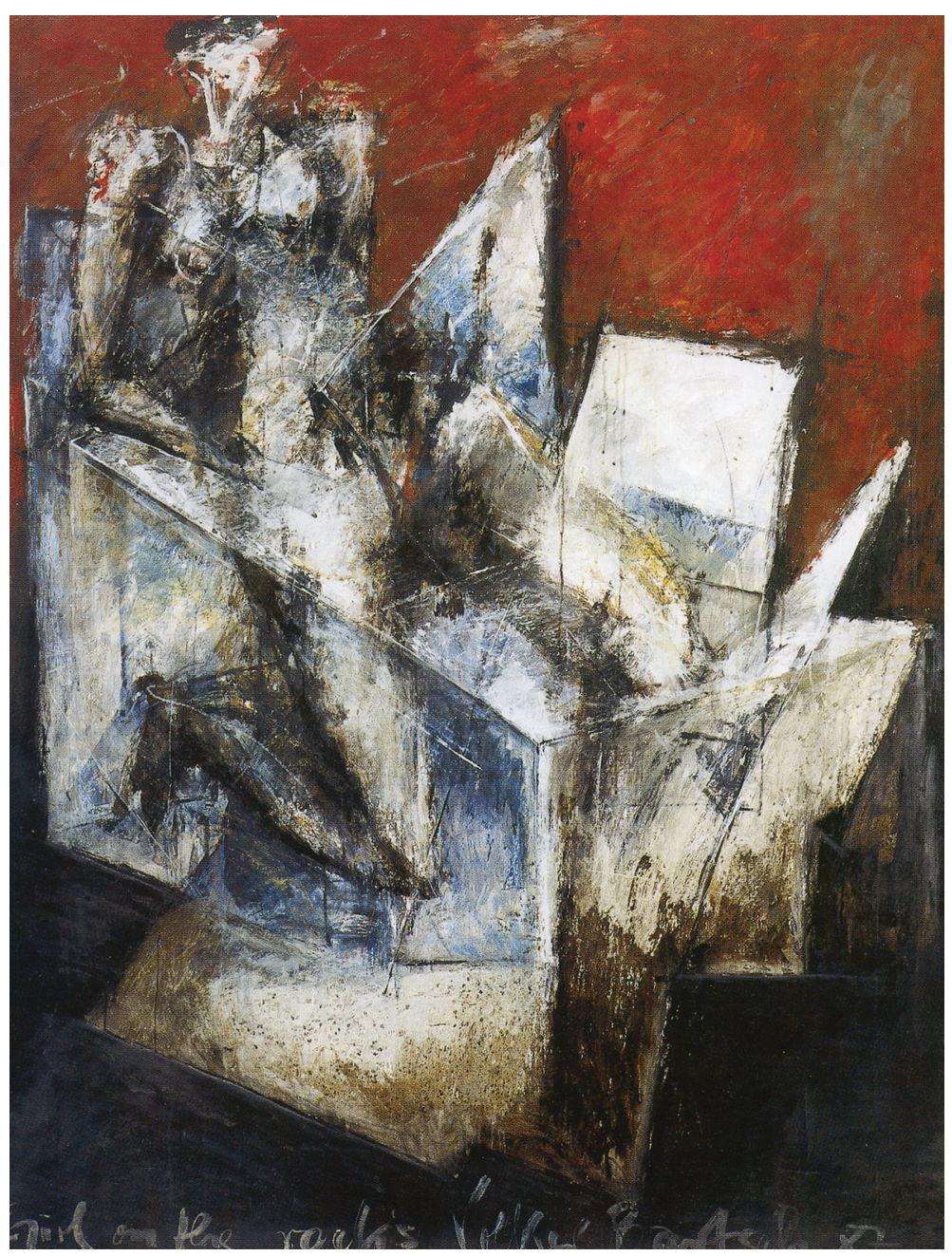

Abb. 1. Volker Bartsch: „Girl on the rocks“ (1987), $180 \times 140 \mathrm{~cm}$, Tempera auf Leinwand.

Seit Anfang 2020 sind alle Staaten der Welt mit der COVID-19-Pandemie befasst. Verkürzt und zugespitzt formuliert, treten die Regierungen und Ministerien der Bevölkerung einerseits als Verkünder von Verordnungen und Einschränkungen sowie als Kontrollinstanzen entgegen, andererseits als Verteiler von finanzieller Unterstützung. Die Regierungen legitimieren ihr Handeln mit dem Hinweis auf die Wissenschaft, deren Vertreter als Berater präsentiert werden und die auch in der öffentlichen Debatte äußerst präsent sind. Die Politik dankt es der Wissenschaft durch Geldzuweisungen für neue Forschungsprojekte. Hier wird eine für die modernen Gesellschaften typische Verschränkung zwischen Wissenschaft und Staat offensichtlich, nämlich in Form eines gegenseitigen Austauschs von Ressourcen, wie dies Mitchel 
G. Ash ausgewiesen hat [8]. Die öffentlichkeitspräsente Rolle der Wissenschaft ist allerdings keineswegs so neu, wie es zunächst scheint.

Ich möchte im Folgenden zwei Punkte historisch behandeln, die jedoch deutliche Anschlussmöglichkeiten an die gegenwärtige Pandemie-Diskussion erlauben. Zum einen geht es um die medizinischen Wissenschaften und deren Spiegelung in der Öffentlichkeit. Zum anderen spreche ich über die soziale und politische Bedeutung von Infektionskrankheiten im Zusammenhang mit besonderen Sinnzuschreibungen und Funktionalisierungen, die öffentlich sichtbar wurden.

\section{Medizinische Wissenschaften und deren Spiegelung in der Öffentlichkeit}

Zunächst zu den Wissenschaften. Die Schrecken der Seuchen haben viele bildliche Darstellungen und Textzeugnisse hervorgebracht. Schon in der frühesten Schrift der abendländischen Literatur wird in der homerischen Ilias (8./7. Jh. v. Chr.) eine Seuche erwähnt. Die Achaier vor Troja werden wegen eines religiösen Frevels von Apoll mit den Pfeilen der Pest bestraft [2]. Der plötzliche massenhafte Tod zeigt die Folgen des Frevels auf.

Mit dem „Schwarzen Tod“ am Ausgang des Mittelalters [9], der zwischen einem Viertel und einem Drittel der europäischen Bevölkerung dahinraffte, kommt die Wissenschaft ins Spiel. 1348 verfasste die Pariser medizinische Fakultät auf Bitten des französischen Königs Philipp VI. ein Gutachten zur Pest. Die Fakultät präsentierte mithilfe von astrologischen Konstellationen und miasmatischen Vorgängen eine Erklärung, die nach dem zeitgenössischen Wissensstand die damalige Pandemie als Ergebnis natürlicher Prozesse erklärte. Irgendwelche moralischen Verfehlungen und göttlichen Bestrafungen wurden nicht als Ursachen erörtert. Doch wurde dieses Gutachten nicht Teil einer öffentlichen Debatte. Die Deutungshoheit über das Geschehen kam damals der Kirche zu, zumal das Leben eines zeitgenössischen Durchschnittsmenschen vollkommen von der Religion geprägt und die Seuche von vielen als Strafe Gottes akzeptiert war. So etwas wie eine bürgerliche Öffentlichkeit, in der die Position der Fakultät hätte Verbreitung und Erörterung finden können, existierte noch nicht.

Dies änderte sich allerdings mit der Aufklärung und dem Entstehen eines modernen Pressewesens im 18. und 19. Jahrhundert. Zunehmend wurden nun wissenschaftliche Inhalte und auch Kontroversen Gegenstand öffentlicher Aufmerksamkeit. Diese Thematisierung hatte teilweise recht unerwartete Reichweiten. So finden wir im Publikationsorgan der Zentralen Kranken- und Sterbekasse der Zimmerer vom 20. August 1896 [10] in einem Artikel über medizinische Versuche an Proletariern einen Absatz über den Tuberkulin-Skandal von 1891.

Robert Koch hatte anlässlich des 10. Internationalen Medizinischen Kongresses im August 1890 das Tuberkulin als ein Heilmittel gegen die Tuberkulose sehr öffentlichkeitswirksam vorgestellt. Die Medien reagierten euphorisch, doch erwies sich bald, dass das Tuberkulin nicht therapeutisch wirksam war, was eine grandiose Niederlage Kochs bedeutete [11]. In dem Absatz in der Zeitschrift „Der Zimmerer“ 
wird darauf verwiesen, dass 1891, als die Tuberkulin-Begeisterung bereits im Schwinden begriffen war und Virchow bereits 27 Todesfälle nach Tuberkulin-Einspritzung festgestellt und auch andere Ärzte in ganz Europa davor gewarnt hatten, dessen ungeachtet in Würzburg noch Versuche an Rekruten mit dem Mittel vorgenommen worden waren. Die Wirkmächtigkeit des Skandals um Kochs vermeintliches Heilmittel und die anschließende Ignoranz der Würzburger Ärzte schaffte es bis in das Organ einer Handwerkergruppe.

Es waren aber nicht nur medizinische Skandale [12], die öffentlich erörtert wurden. Die Bemühungen von Friedrich Loeffler um die Entwicklung einer Immunisierungsmethode gegen die Maul- und Klauenseuche wurden ebenfalls von den Medien begleitet. Die zentralen Fragen waren damals wie heute, wann die Immunität eintreten und wie lange sie bestehen bleiben würde. 1898 konnte Loeffler in verschiedenen Feldversuchen erste Erfolge verzeichnen. Dennoch blieb in der Öffentlichkeit die Skepsis gegenüber dieser Methode auch in den folgenden Jahren weit verbreitet.

Am 27. April 1911 erschien in der Berliner Abendpost ein Artikel, in dem unter Berufung auf zwei prominente Veterinärmediziner Loefflers Versuche heftig kritisiert wurden. Das Vertrauen auf Loefflers Methoden, auch seitens des preußischen Landwirtschaftsministeriums, und die nicht konsequente Anwendung von restriktiven Schutzmaßnahmen habe zu einer Verbreitung der Seuche geführt. Hinter diesen Angriffen stand eine Fraktion von Veterinärmedizinern, die auf die Methoden der Quarantäne und Keulung setzten und der modernen Immunisierung und Impfung starke Vorbehalte entgegenbrachten [13].

Diese modernen Verfahren jedoch standen für einen medizinischen Weg, bürgerliche und ökonomische Freiheiten, wie die des Reisens und des Warenaustauschs, zu gewährleisten. In den Tageszeitungen wurden Auseinandersetzungen zwischen konservativen und modernen Ansätzen in der Seuchenbekämpfung ausgetragen. Die Öffentlichkeit war also schon vor 150 Jahren in wissenschaftliche und gesundheitspolitische Kontroversen einbezogen.

Auch heute ist medizinische Wissenschaft in der Öffentlichkeit mehr denn je präsent. Wir finden einerseits seriöse Aufklärungsformate, deren Reichweite jedoch begrenzt ist. Andererseits werden über Personalisierungen und die Betonung von wissenschaftlichen Differenzen eher Inszenierungen von wissenschaftlichen Wettkämpfen präsentiert. Wissenschaft fungiert hier in Zeiten des Lockdowns als Sportersatz und füllt die Lücke, die fehlende Sportwettkämpfe reißen. So findet sich am 4. April 2020 in der „Berliner Zeitung“ ein Beitrag mit der Überschrift: „Welchem Experten vertrauen Sie am meisten? B.Z. stellt unsere wichtigsten Kämpfer gegen Corona vor." Nach einer Umfrage, an der sich 60.000 Leserinnen und Lesern beteiligten, wurde ein Ranking der in der Öffentlichkeit bekannten Forscherinnen und Forscher präsentiert. In dem Beitrag werden die sogenannten wichtigsten Thesen der Gelisteten beschrieben und auch der größte Irrtum jedes Einzelnen aufgeführt [14]. Dieses Format erinnert an die Bewertung von Sportlern nach ihren Stärken und Schwächen. Solche Beispiele sind allerdings kaum geeignet, um klarzumachen, was Wissenschaft ist beziehungsweise was sie leisten kann und was nicht. Die gegenwärtig viel beschworene positive Präsenz der Wissenschaften in den Medien scheint in Teilen recht fragil. 


\section{Soziale und politische Bedeutung von Infektionskrankheiten}

Im Zusammenhang mit Sinnzuschreibungen an Seuchen und deren Funktionalisierungen möchte ich drei Bereiche abgrenzen: 1) für das Individuum, 2) für die Gesellschaft und 3) für den Staat beziehungsweise das politische System.

Ein Beispiel aus der Literatur, das der Tuberkulose eine besonders sinnstiftende Bedeutung zuweist, findet sich im „Zauberberg“ von Thomas Mann. Für einen jungen, mediokren Ingenieur aus Hamburg wird die Auseinandersetzung mit der Tuberkulose in der Atmosphäre eines Sanatoriums zum Ausgangspunkt einer Zeitreise, die in die Welt der Politik und Philosophie, in die Konfrontation mit Krankheit, Tod und Liebe führt. Es ist letztlich die Tuberkulose, die die geistige Beschränktheit des jungen Mannes aufzulösen vermag. Sie erhält so eine überraschend positive Konnotation. Auf die medizinische Kritik an seinem Werk antwortete Thomas Mann, dass er seine Gestalten in einer hermetischen Retorte angesiedelt habe, in der ein schlichter Stoff zu ungeahnter ideeller Veredelung empor gezwängt und geläutert werde. Die Konfrontation mit der Tuberkulose und ihren vielfältigen Erscheinungen ermöglichte einem jungen Mann Grenzerfahrungen, die sein Selbst erweiterten. Die Reichweite solcher sinnstiftenden narrativen Konstruktionen war allerdings auf das Bildungsbürgertum als lesende Gruppe beschränkt und unterscheidet sich auch deutlich von anderen Lesarten der Tuberkulose.

In der gesellschaftlichen Debatte der gegenwärtigen Pandemie wird gelegentlich dem Lockdown eine hohe sinnstiftende Bedeutung zugewiesen. Demnach hat er im Hinblick auf die gefährlichen Konsequenzen des Klimawandels gewissermaßen das Modell einer zukünftigen Gestaltung des Lebens auf der Erde paradigmatisch antizipiert. Entschleunigung, weniger Verkehr und Konsum waren lebbar und konnten den Praxistest bestehen. Welchen Preis dies für die unterschiedlich betroffenen sozialen Gruppen und Länder hat, wird dabei vielfach übersehen.

Die Geschichte bietet zahlreiche Beispiele dafür, wie Seuchen als Möglichkeiten der Selbstvergewisserung fungierten, der Abgrenzung des „Wir“ von den „Anderen“, den gefahrbringenden Fremden - nicht selten verbunden mit Justierungen von Moral- und Wertvorstellungen und Menschenbildern. Fast immer wird dabei den Anderen auch so etwas wie Schuld an den sozialen und ökonomischen Verwerfungen zugewiesen, die durch das Infektionsgeschehen hervorgerufen wurden [15].

Der „Schwarze Tod“, die Pestpandemie am Ende des Mittelalters (1347-1351), galt zwar einerseits als Strafe Gottes, andererseits wurden Juden und Leprakranke als Brunnenvergifter für das Geschehen verantwortlich gemacht. Die Juden und Leprösen als Sündenböcke waren die bösen Anderen, und die sozialen Spannungen, die durch die Seuche verschärft wurden, entluden sich in massenhaften Pogromen. Die Seuche förderte mithilfe einer Markierung, Ausgrenzung und Tötung der bösen Anderen die Selbststilisierung eines ,guten Wir“, der unrechtmäßig Bedrohten.

Nicht immer muss die Markierung der Anderen mit solchen brutalen Konsequenzen verbunden sein. Aber Schuldzuweisungen an bestimmte Gruppen in der Bevölkerung, die man für eine weitere Verbreitung der Infektion verantwortlich macht, sind auch im 20. und 21. Jahrhundert weit verbreitet. So war zum Beispiel am 
27. Oktober 1918 anlässlich der Grippepandemie [16, 17] im „Hamburger Fremdenblatt“ als Erklärung für die vielen erkrankten Frauen zu lesen: Es seien ,gegenwärtig Frauen und Mädchen, die überwiegend den Besuch der Lichtspieltheater bestreiten; der Grippe-Bazillus findet aber gerade in der Dunkelheit der Lichtspieltheater geeigneten Nähr- und Verbreitungsboden“ [18]. Der Subtext dieser Passage transportiert die Aussage, dass moralisch verwerfliche Besuche in zweifelhaften dunklen Etablissements die Verbreitung der Grippe unter den sich falsch verhaltenden Frauen fördere und diese zu „Superspreadern“ mache.

Die Abgrenzung zu den gefährlichen Anderen ist vielfach mit räumlichen Zuweisungen und allen möglichen Grenzziehungen verbunden. Dabei zeigt sich eine beeindruckende Geschwindigkeit in der Neudefinition der verschiedenen Gefahrenräume. In der gegenwärtigen Pandemie war es zunächst das weit entfernte China, doch kam die Bedrohung immer näher, bis es die im eigenen Land ganz nahe gelegenen Hotspots waren, von denen die tödliche Gefahr ausging. Die angeordneten Abschottungen gehören in das Seuchenbekämpfungsrepertoire des 17. Jahrhunderts [19], wobei verschiedentlich in Medien und Politik alte Stereotype über die Stadt als unkontrollierbares Sünden- und Seuchenbabel bedient werden.

Michel Foucault [20] sah in den Seuchen die Geburtshelfer der modernen Staaten, weil sie den Staaten die Möglichkeit boten, sich als Disziplinierungsmacht auszuweisen, indem sie ihre Regierungstechniken im Kampf gegen Pest und Cholera ausprobierten. Der Kampf gegen Ansteckung markiert die Urszene der Disziplinargesellschaft: Die Menschen werden separiert, parzelliert, beobachtet und kontrolliert. Man wird in Privaträume und auf sich selbst zurückgeworfen, die Reproduktion wird gegenüber der Produktion in den Vordergrund gerückt. Die staatlich vorangetriebene Seuchenbekämpfung fungierte in der Moderne immer wieder als Gradmesser für die staatliche Leistungsfähigkeit und für die Überlegenheit des jeweiligen politischen Systems. Seit gut 200 Jahren dienen dabei auch Statistiken über Erkrankung und Todeszahlen als bewährte Erfolgsbelege in der Auseinandersetzung der Nationen und Systeme.

Die Bekämpfung von Seuchen wird auch gegenwärtig im Kampf der politischen Systeme instrumentalisiert. Anfang Februar gingen die Bilder des Krankenhauses mit 1000 Betten um die Welt, das in Wuhan (VR China) in nur zehn Tagen errichtet worden war. Manche Menschen in Deutschland waren von dieser Leistung sehr angetan und fragten, ob man nicht auch bei uns die demokratischen Verfahrensweisen im Interesse einer schnellen und wirksamen Pandemiebekämpfung etwas zurückfahren könne. In der medialen Auseinandersetzung der politischen Systeme markierte der Bau des ZehnTage-Krankenhauses einen Triumph des autokratischen, nicht demokratischen Staates über die demokratischen, partizipatorischen westlichen Gesellschaften. Mittlerweile bestärken die lückenlosen Alltagsüberwachung mithilfe moderner Mobilgräte in verschiedenen Staaten diese demokratieskeptischen Haltungen.

\section{Fazit}

Die präsentierten Zeugnisse und Beispiele sind Ausdruck einer medialen und kulturellen Auseinandersetzung von Gesellschaften mit Seuchen. Dabei offenbart sich, dass bestimmte Reaktionsmuster und Zuschreibungen schon seit Jahrhunderten 
existieren, wenn auch wegen der zeitgenössischen technologischen und kulturellen Gegebenheiten das Erscheinungsbild dieser Reaktionen different gestaltet ist. Es können so anthropologische und soziale Konstanten in verschiedensten Gesellschaften ausgemacht werden, wie zum Beispiel Sinnzuweisungen, Funktionalisierungen, Identitätsversicherung, Diskussion von Moralvorstellungen oder Suche nach Grenzerfahrung. Kulturelle Betätigungen und die Befassung mit künstlerischen Produkten gehen weit über Freizeitgestaltungen hinaus; sie verweisen auf ein besonderes Feld gesellschaftlicher Aktivitäten, durch die verschiedenartigste Phänomene im Kontext von Seuchen aufgegriffen, verarbeitet und in ein historisch gewachsenes Korpus von Erfahrungen und Wissensbeständen eingeschrieben wurden.

Wenn aber eine primär kommunikative Erlebbarkeit eines die Öffentlichkeit dominierenden Infektionsgeschehens vorherrscht, so besteht die Gefahr, dass diese nicht mehr als biologische Realität, sondern als ein Phänomen aufgefasst wird, das primär in der kommunikativen Sphäre existiert. In diesen kommunikativen Prozessen kann leicht die eigene Meinung über eine Nichtexistenz einer Pandemie verfestigt werden. Ein Verstehen der kulturellen, medialen und kommunikativen Vielfalt, die Gesellschaften im Umgang mit Pandemien in Vergangenheit und Gegenwart schufen und aufweisen, kann helfen, den komplexen Herausforderungen von Pandemien gerecht zu werden.

\section{Literatur}

1. Dinges, M.: Neue Wege in der Seuchengeschichte? In: Dinges, M., Schlich T. (Hrsg.) Neue Wege in der Seuchengeschichte (Medizin, Gesellschaft und Geschichte, Beiheft 6), S. 7-24. Stuttgart (1995)

2. Leven, K.H.: Die Geschichte der Infektionskrankheiten. Von der Antike bis ins 20. Jahrhundert. Landsberg/Lech (1997)

3. Briese, O.: Angst in Zeiten der Cholera. Das schlechte Gedicht. Strategien literarischer Immunisierung, Seuchen-Cordon I. Berlin (2003)

4. Winkle, S.: Geißeln der Menschheit. Kulturgeschichte der Seuchen, 3. Aufl. Düsseldorf (2005)

5. Schmiedebach, H.-P.: Seuchen und ihre Spuren in Gesellschaft, Kultur und Politik. In: Fangerau, I., Polanski, J. (Hrsg.) Medizin im Spiegel ihrer Geschichte, Theorie und Ethik. Schlüsselthemen für ein junges Querschnittsfach (Kultur Anamnesen, 4), S. 235-257. Stuttgart (2012)

6. Thießen, M. (Hrsg.) Infiziertes Europa. Seuchen im langen 20. Jahrhundert. Berlin (2014)

7. Snowden, F. M.: Epidemics and Society. From the Black Death to the Present. New Haven (2020)

8. Ash, M. G.: Wissenschaft und Politik als Ressourcen für einander. In: vom Bruch, R., Kaderas, B. (Hrsg.) Wissenschaften und Wissenschaftspolitik. Bestandsaufnahmen zu Formationen, Brüchen und Kontinuitäten im Deutschland des 20. Jahrhunderts, S. 32-51. Stuttgart (2002)

9. Bergdolt, K.: Der schwarze Tod in Europa. Die Große Pest und das Ende des Mittelalters. München (1994)

10. Anonym (Dr.-r): Proletarier als Versuchsthiere. In: Der Zimmerer, Publikationsorgan der Zentralen Kranken- und Sterbekasse der Zimmerer 8, Nr. 35, S. 2-4 (20. August 1896) 
11. Gradmann, C.: Krankheit im Labor. Robert Koch und die medizinische Bakteriologie. Göttingen (2005)

12. Labisch, A.: „Skandalisierte Krankheiten“ und „echte Killer“ - Zur Wahrnehmung von Krankheiten in Presse und Öffentlichkeit. In: Andel, M. et al. (Hrsg.) Propaganda, (Selbst-) Zensur, Sensation. Grenzen von Presse- und Wirtschaftsfreiheit in Deutschland und Tschechien seit 1871, S. 273-289. Essen (2005)

13. Schmiedebach, H.-P.: The Prussian State an microbiological research - Friedrich Loeffler and his approach to the ,invisible“ virus. Arch. Virol. 15, 9-23 (1999)

14. Anonym: Welchem Experten vertrauen Sie am meisten? B.Z stellt unsere wichtigsten Kämpfer gegen Corona vor. In: Berliner Zeitung, S. 8 (4. April 2020)

15. Fangerau, H., Labisch, A.: Pest und Corona. Pandemien in Geschichte, Gegenwart und Zukunft. Freiburg im Breisgau (2020)

16. Michels, E.: Die „Spanische Grippe“ 1818/19. Verlauf, Folgen und Deutungen in Deutschland im Kontext des Ersten Weltkriegs. In: Vierteljahreshefte für Zeitgeschichte, S. 1-33, 58 (2010)

17. Witte, W.: Tollkirschen und Quarantäne. Die Geschichte der Spanischen Grippe. Berlin (2010)

18. Thießen, M.: Infizierte Gesellschaften: Sozial und Kulturgeschichte von Seuchen. In: Aus Politik und Zeitgeschichte 65 H. 20/21, S. 11-18 (2015)

19. Leven, K. H.: Die Welt mit und nach Corona: medizinhistorische Perspektiven. In: Kortmann, B., Schmidt, G.G. (Hrsg.) Jenseits von Corona. Unsere Welt nach der Pandemie - Perspektiven aus der Wissenschaft, S. 91-98. Bielefeld (2020)

20. Foucault, M.: Überwachen und Strafen. Die Geburt des Gefängnisses. Frankfurt a. M. (1994)

Open Access Dieses Kapitel wird unter der Creative Commons Namensnennung - Nicht kommerziell - Keine Bearbeitung 4.0 International Lizenz (http://creativecommons.org/ licenses/by-nc-nd/4.0/deed.de) veröffentlicht, welche die nicht-kommerzielle Nutzung, Vervielfältigung, Verbreitung und Wiedergabe in jeglichem Medium und Format erlaubt, sofern Sie den/die ursprünglichen Autor(en) und die Quelle ordnungsgemäß nennen, einen Link zur Creative Commons Lizenz beifügen und angeben, ob Änderungen vorgenommen wurden. Die Lizenz gibt Ihnen nicht das Recht, bearbeitete oder sonst wie umgestaltete Fassungen dieses Werkes zu verbreiten oder öffentlich wiederzugeben.

Die in diesem Kapitel enthaltenen Bilder und sonstiges Drittmaterial unterliegen ebenfalls der genannten Creative Commons Lizenz, sofern sich aus der Abbildungslegende nichts anderes ergibt. Sofern das betreffende Material nicht unter der genannten Creative Commons Lizenz steht und die betreffende Handlung nicht nach gesetzlichen Vorschriften erlaubt ist, ist auch für die oben aufgeführten nicht-kommerziellen Weiterverwendungen des Materials die Einwilligung des jeweiligen Rechteinhabers einzuholen. 